\title{
Neurofilament protein in cerebrospinal fluid: a potential marker of activity in multiple sclerosis
}

\author{
J N Lycke, J-E Karlsson, O Andersen, L E Rosengren
}

\begin{abstract}
The neurofilament protein is a major structural protein of neurons and a marker for axonal damage. The concentrations of the light subunit of the neurofilament triplet protein (NFL) in CSF were significantly increased in patients with relapsing-remitting multiple sclerosis compared with healthy controls $(p<0.001)$. Seventy eight per cent of patients with multiple sclerosis showed increased NFL concentrations. Significant correlations between the NFL concentration in CSF and clinical indices were discerned for disability, exacerbation rate, and time from the start of the previous exacerbation to the time of the lumbar puncture. The results suggest that axonal damage occurs during relapsingremitting multiple sclerosis and that the damage contributes to disability and the appearance of clinical exacerbations. The concentration of NFL in CSF is a potential marker of disease activity in multiple sclerosis and might be useful in future clinical trials of multiple sclerosis.
\end{abstract}

Neuroscience, Department of Neurology, Göteborg University,

Sahlgrenska University

Hospital, S-413 45

Göteborg, Sweden

J Lycke

O Andersen

Institute of Anatomy and Cell Biology,

Göteborg University, S-413 45 Göteborg,

Sweden

J-E Karlsson

L E Rosengren

Correspondence to: Dr Jan Lycke, Institute of Clinical Neuroscience,

Department of Neurology, Göteborg University, Sahlgrenska University Hospital, S-413 45 Göteborg, Sweden.

Telephone 004631603210 ; fax 004631602467 .

Received 31 January 1997 and in final revised form 22 August 1997

Accepted 29 August 1997 of neurological deficiency, augmented astrogliosis, and axonal loss. ${ }^{6}$ However, there are indications of axonal loss also in early multiple sclerosis with a relapsing-remitting course, ${ }^{7}$ and in recent years investigations with magnetic resonance spectroscopy $(\mathrm{MRS})^{8}$ and magnetisation transfer ratio ${ }^{9}$ suggest that neural damage occurs, not only in chronic multiple sclerosis lesions, but also in acute lesions. In this study we determined the concentration of NFL in CSF from patients with relapsingremitting multiple sclerosis and investigated if the concentration was influenced by clinical signs of disease activity.

\section{Materials and methods}

Sixty patients (19-45, median 33 years) with relapsing-remitting clinically definite multiple sclerosis, ${ }^{10}$ with a mean duration of multiple sclerosis of 5.8 years (0.4-28 years) and at least two exacerbations within the previous two year period and a neurological deficit score $<4$ on the expanded disability status scale (EDSS), ${ }^{11}$ were treated in a two year, double blind clinical trial with oral acyclovir, (800 $\mathrm{mg} \times 3)$, or placebo to test the hypothesis of a possible involvement of herpesviruses in the aetiopathogenesis of multiple sclerosis. ${ }^{12}$ No immunomodulatory treatment was given before or during the trial.

Patients were in remission without exacerbations for a period of at least one month before the start of the trial. The exacerbation rate and the disability, scored with EDSS, served as primary and secondary objectives for the acyclovir trial. Thirty nine patients had a duration of disease of at least two years before the trial - that is, they possessed a prestudy exacerbation rate baseline. Eleven healthy subjects (28-64, median 33 years) without history of neurological disorder and with a normal neurological examination served as controls in the present study. The study was approved by the ethics committee of the University of Göteborg, Sweden, and patients and controls signed an informed consent form.

Samples of CSF were obtained by lumbar puncture and fractions of CSF were stored in $0.5 \mathrm{ml}$ aliquots at $-80^{\circ} \mathrm{C}$. Analysis of NFL in CSF was performed according to a previously described sandwich enzyme linked immunosorbent assay (ELISA). ${ }^{4}$ The standard curve ranged from 125 to $16000 \mathrm{ng} / \mathrm{l}$. The sensitivity 


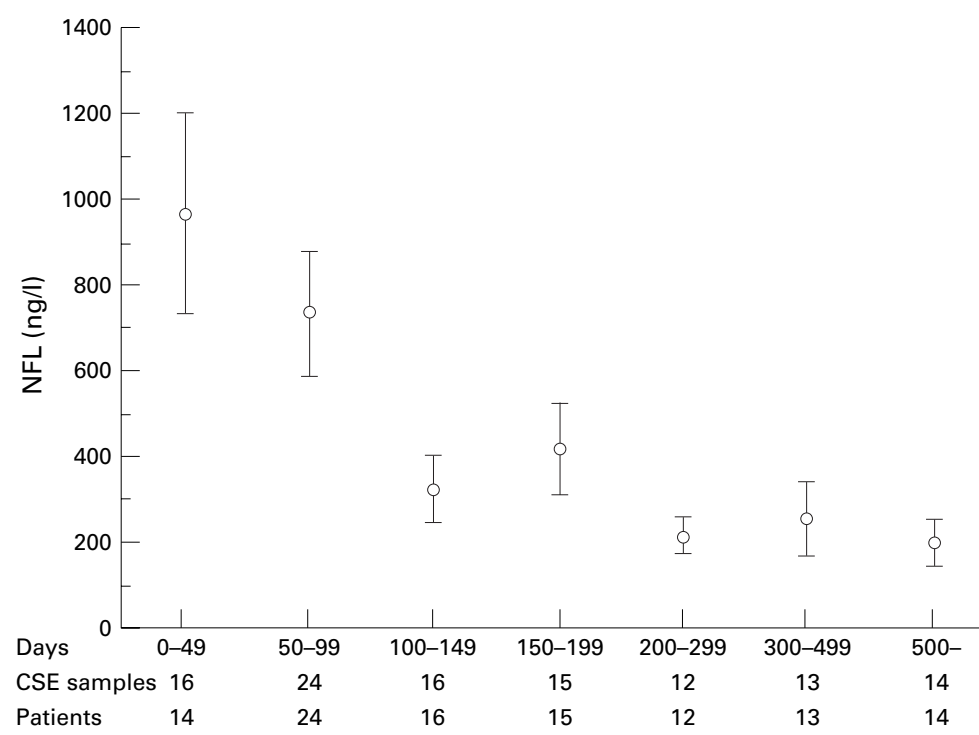

Mean NFL concentrations ( $n g / l$ ) in CSF related to the length of time (days) from the start of the previous clinical exacerbation to the time of the lumbar puncture. Sampling of CSF was performed once $(n=10)$ or twice $(n=50)$ from 60 patients with multiple sclerosis. Bars indicate \pm 1 SEM.

of the assay was $125 \mathrm{ng} / \mathrm{l}$. The CSF sampling was performed in patients at inclusion and two years later at termination of the acyclovir or placebo treatment, and once in controls. In one patient the pretreatment CSF sample was missing and in five patients the CSF sample at treatment termination was missing. In total 59 pretreatment and 55 post-treatment CSF samples constituted 54 pairs and six single CSF samples from the multiple sclerosis population. The influence of exacerbations on the NFL concentration was determined only in patients with a relapsing-remitting course-patients who turned into a progressive course $(n=4)$ during the trial were excluded from these analyses.

The Mann-Whitney $U$ test, corrected for ties, was used to compare NFL concentrations between groups and the Wilcoxon signed rank test, corrected for ties, was used to compare changes of NFL concentrations over time within groups. Spearman's rank correlation coefficient, corrected for ties, was used to discern relations beween the NFL concentration and different clinical and demographic variables.

\section{Results}

The median pretreatment NFL concentration in patients with multiple sclerosis was $265 \mathrm{ng} / \mathrm{l}$ and was below the limit of detection for the NFL assay $(125 \mathrm{ng} / \mathrm{l})^{4}$ in all controls $(\mathrm{p}<0.001)$. Increased NFL concentrations $(>125 \mathrm{ng} / \mathrm{l})$ were detected at least once in 47 $(78 \%)$ patients with multiple sclerosis. Two years of acyclovir treatment did not influence the NFL concentration in the CSF. Individual differences between pretreatment and posttreatment NFL concentrations showed equal distributions between the acyclovir and placebo group. Four patients (three acyclovir and one placebo treatment) turned into a progressive course during the trial. Although the
EDSS score increased by a median of 2 (range 0.5-3.0) during progression in these patients, an obvious increase of the NFL concentration was found in only one of them.

There was a moderate relation between the NFL concentration and the EDSS score before treatment $\left(\mathrm{r}_{\mathrm{s}}=0.27, \mathrm{p}<0.05\right)$ as well as two years later at treatment termination $\left(r_{s}=0.34\right.$, $\mathrm{p}<0.01)$. A relation was also disclosed between the NFL concentration at treatment termination and the exacerbation rate during the two year trial $\left(r_{s}=0.38, p<0.01\right)$. This relation was stronger in the corresponding analysis for the two year pre-treatment period $\left(r_{s}=0.56\right.$, $\mathrm{p}<0.001$ ) including only patients with a two year exacerbation rate baseline.

The NFL concentration was higher in CSF samples obtained during the first two to three months after the start of the previous exacerbation and dropped gradually thereafter to a low level (figure). In patients with a relapsingremitting course throughout the trial the NFL concentration at treatment termination correlated with the length of time from the start of the previous exacerbation to the time of the lumbar puncture $\left(r_{s}=-0.48, p<0.001\right)$. A similar relation was also shown for the pretreatment period $\left(r_{s}=-0.34, p<0.01\right)$. The NFL concentration showed no relation with age, sex, or duration of multiple sclerosis. Moreover, there was no correlation between the NFL concentration in CSF and the blood-brain barrier permeability, monitored by the CSF:serum albumin ratio $\left(r_{s}=-0.05, p=0.6\right)$. Hence, although changes in the blood-brain barrier might influence the NFL concentration in CSF the degree of influence seemed to be negligible.

\section{Discussion}

NFL is the light subunit of the neurofilament triplet protein. It is a major structural element of neurons. It constitutes only a minor part of the cytoskeletal constituents in the neuronal cell body and dendrites relative to axons. ${ }^{1}$ Hence, increased concentrations of NFL in CSF mainly reflects damage to the axonal compartment. The present study shows that NFL is significantly increased in the CSF of patients with relapsing-remitting multiple sclerosis. High NFL concentrations were related to clinical exacerbations, the exacerbation frequency, and the degree of disability.

During recent years supplementary markers for multiple sclerosis disease activity have been developed. Biological markers in serum and CSF have mainly been focused on the immunological activity of multiple sclerosis. ${ }^{13} 14$ However, the results from these studies have been contradictory and none of these markers have yet been established as a disease activity marker. Although the use of MRI of the brain has become undisputed when new treatments for multiple sclerosis are evaluated, MRI tell us little about the neuropathological differentiation of multiple sclerosis lesions. Magnetic resonance spectroscopy may add information about this issue, ${ }^{8}$ but the access to MRS is limited. Hence, disease activity markers in serum and CSF which add information about the neuropathological changes in multiple sclerosis 
are of value. We have previously shown that glial fibrillary acidic protein, a marker for astrogliosis, is increased in multiple sclerosis and is correlated with disability. ${ }^{15}$ The present study shows that NFL determinations in CSF may be a marker for axonal damage during relapsing-remitting multiple sclerosis.

The fact that active multiple sclerosis lesions on MRI of the brain are five to 10 times more frequent than the number of clinical exacerbations has previously been explained by the relatively low number of lesions in clinically eloquent areas and that some clinically active lesions are located in the spinal cord. However, an alternative explanation could be that lesions which cause axonal damage are more likely to cause clinical symptoms. The present study supports this hypothesis as high NFL concentrations were associated with clinical exacerbations and low concentrations with clinically stable periods. Recent studies with $\mathrm{MRS}^{8}$ and magnetisation transfer ratio ${ }^{9}$ also indicate that axonal damage occurs in acute multiple sclerosis lesions.

In conclusion, NFL determination in CSF is a potential CSF marker of disease activity in relapsing-remitting multiple sclerosis. Our results suggest that axonal damage occurs during relapsing-remitting multiple sclerosis and that the damage contributes to disability and the appearance of clinical exacerbations. Further investigations are needed to discernif determinations of CSF in NFL are useful as an outcome measure in multiple sclerosis trials.

This work was supported by grants from the NHR Foundation, the Hjalmar Svenssons Foundation, the Lundbeck Foundation, and the Edith Jacobsson Foundation.
1 Lasek RJ. Studying the intrinsic determinants of neuronal form and function. In: Lasek RJ, Black MM, ed. Intrinsic determinants of neuronal form and function. New York: Allan R Liss, 1988.

2 Karlsson J-E, Rosengren LE, Haglid KG. Quantitative and qualitative alterations of neuronal and glial intermediate filaments in rat nervous system after exposure to 2,5hexanedione. 7 Neurochem 1991;57:1437-44.

3 Wang S, Lees GJ, Rosengren LE, et al. The effect of an N-methyl-D-aspartate lesion in the hippocampus on glial and neuronal marker proteins. Brain Res 1991;541: 334-41.

4 Rosengren L, Karlsson J-E, Karlsson J-O, et al. Patients with amyotrophic lateral sclerosis and other neurodegenerative diseases have increased levels of neurofilament protein in CSF. 7 Neurochem 1996;67:2013-8.

5 Adams CWM. The onset and progression of the lesion in multiple sclerosis. F Neurol Sci 1975;25:165-82.

6 Barnes D, Munro PMG, Youl BD, et al. The longstanding MS lesion. A quantitative MRI and electron microscopic study. Brain 1991;114:1271-80.

7 MacFadyen DJ, Drance SM, Douglas GR, et al. The retinal nerve fiber layer, neuroretinal rim area, and visual evoked potentials in MS. Neurology 1988;38:1353-8.

8 Davie CA, Hawkins CP, Barker GJ, et al. Serial proton magnetic resonance spectroscopy in acute multiple sclerosis lesions. Brain 1994;117:49-58.

9 Gass A, Barker GJ, Kidd D, et al. Correlation of magnetization transfer ratio with clinical disability in multiple sclerosis. Ann Neurol 1994;36:62-7.

10 Poser CM, Paty DW, Scheinberg L, et al. New diagnostic criteria for multiple sclerosis: guidelines for research protocols. Ann Neurol 1983;13:227-31.

11 Kurtzke JF. Rating neurologic impairment in multiple sclerosis: an expanded disability status scale (EDSS). Neurology 1983;33:1444-52.

12 Lycke J, Svennerholm B, Hjelmquist E, et al. Acyclovir treatment of relapsing-remitting multiple sclerosis. A randomized, placebo-controlled, double-blind study. I Neurol 1996;243:214-24

13 Rieckhmann P, Martin S, Weichselbraun I, et al. Serial analysis of circulating adhesion molecules and TNF receptor in serum from patients with multiple sclerosis: cICAM-1 is an indicator for relapse. Neurology 1994;44: 2367-72.

14 Shaw CE, Dunbar PR, Macaulay HA, et al. Measurement of immune markers in serum and cerebrospinal fluid of multiple sclerosis patients during clinical remission. 7 Neurol 1995;242:53-8.

15 Rosengren LE, Lycke J, Andersen O. Glial fibrillary acidic protein in CSF of multiple sclerosis patients: relation to neurological deficit. $\mathcal{F}$ Neurol Sci 1995;133:61-5. 